\section{Sistemas de protección social para el adulto mayor en América Latina y el Caribe}

\author{
Rubén Suárez ${ }^{1}$ \\ y Claudia Pescetto ${ }^{1}$
}

La transición demográfica que viven los países de América Latina y el Caribe, caracterizada por el rápido crecimiento del número de adultos mayores, impone numerosos desafíos a los encargados de tomar las decisiones y de diseñar las políticas orientadas a satisfacer las necesidades de salud y de consumo de las personas de mayor edad. En este documento se describen de forma sintética los procesos de transición demográfica y de envejecimiento de la población en la Región, especialmente en América Latina y el Caribe, se exploran algunas implicaciones económicas de estos procesos y se evalúa el alcance actual de la cobertura de prestaciones de los pilares de la protección social y del ahorro para la vejez. Además, se analizan algunos de los elementos que se deben tener en cuenta al elaborar políticas orientadas a garantizar —al menos - niveles mínimos de calidad de vida y el acceso a los servicios de salud para la creciente población de adultos mayores en América Latina.

\section{ENVEJECIMIENTO DE LA POBLACIÓN EN AMÉRICA LATINA Y EL CARIBE}

\section{Transición demográfica y envejecimiento}

El mejoramiento de las condiciones de vida de la población y la mayor disponibilidad de servicios de salud han conducido a una transición demográfica acelerada en América Latina y el Caribe. Esta transición se caracteriza por la reducción de las tasas de fecundidad y de mortalidad, lo que modifica la estructura por edades de la población. Como resultado, en las dos últimas décadas la población de América Latina ha empezado a experimentar un proceso de "envejecimiento". Si bien en 1975 la población mayor de 60 años en esta parte de la Región era de 21 millones de personas $(6,5 \%$ de la población total), en solo 25 años este grupo de población ascendió a 41 millones de personas $(8,1 \%$ de la población). Según proyecciones demográficas de la Comisión Económica para América Latina y el Caribe (CEPAL), en las próximas décadas aumentarán aun más las tasas de crecimiento de la población mayor de 60 años de edad (1), de manera que en 2025 este grupo de edad será de alrededor de 100 millones de personas y en 2050, de cerca de $200 \mathrm{mi}-$ llones. En consecuencia, los adultos mayores de 60 años que representaban el $8,1 \%$ de la población total de América Latina en el año 2000, pasarán a 
ser el 14,1\% en 2025 y representarán cerca de la cuarta parte de la población total (23,4\%) en 2050.

Estos cambios en los patrones de crecimiento de los distintos grupos de edad de la población son un reflejo de la transición demográfica y tendrán un impacto importante sobre la tasa de dependencia económica de los adultos mayores, es decir, la relación entre el número de personas mayores de 60 años y la población en edad laboral (personas entre 15 y 59 años de edad).

\section{Ventana de oportunidades: ¿todavía abierta en América Latina y el Caribe?}

Durante el proceso de transición demográfica se presenta una "ventana de oportunidades" durante la cual se debe observar una reducción en la tasa total de dependencia económica. Este período, de duración relativamente corta, ofrece la oportunidad de incrementar los niveles de ahorro para la vejez o el nivel de transferencias por persona dependiente, mientras se mantiene constante el ingreso disponible por persona en edad laboral. Esta reducción del número de personas dependientes debe dar como resultado el incremento del ingreso per cápita de la población.

El cambio acelerado en la estructura por edades de la población en América Latina y el Caribe entre 1950 y 2000 y las proyecciones para los próximos 50 años indican que esta "ventana de oportunidades" se cerrará en los próximos 20 años.

\section{Indicadores demográficos de dependencia}

En los próximos 50 años, el número de personas en edad laboral por cada persona en edad de retiro - es decir, el inverso de la tasa de dependencia- en América Latina y el Caribe se reducirá de 7,6 en el año 2000 a 4,3 en 2025 y a tan solo 2,5 en el año 2050 (figura 1). El envejecimiento de la población en los países del Caribe de habla inglesa es más grave que el esperado en los de América Latina, pues para el año 2025 la tasa de dependencia en esos países caribeños será similar a la de Europa en el año 2000 (alrededor de 0,30). Esto quiere decir que habrá solamente 3,3 personas en edad laboral por cada persona en edad de retiro, mientras que para el año 2050 se espera que haya solamente dos personas en edad laboral por cada persona mayor de 60 años en esos países.

A la transición demográfica descrita se suma la transición epidemiológica, caracterizada por el incremento en la incidencia y prevalencia de las enfermedades que se presentan más frecuentemente en los adultos mayores. Los cambios en el patrón de
FIGURA 1. Personas entre 15 y 49 años de edad por cada adulto mayor de 60 años. Años 2000-2050

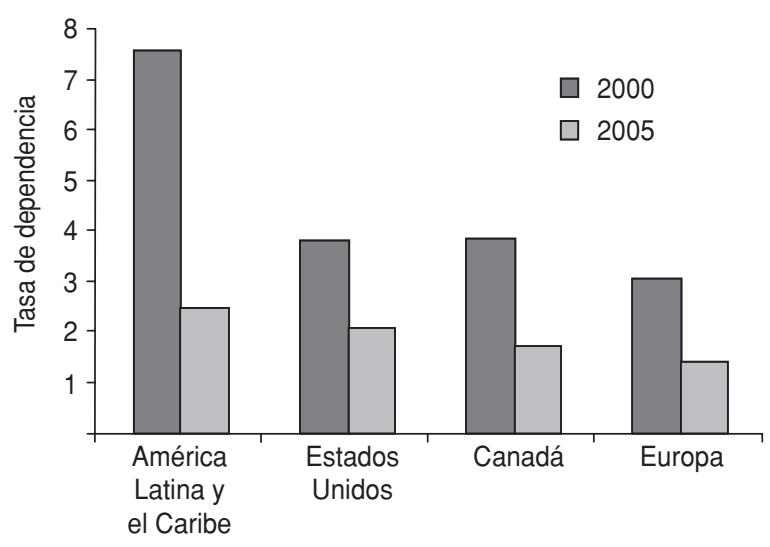

Fuente: CEPAL (2).

enfermedades asociados con el envejecimiento de la población permiten prever un aumento en la demanda de los recursos financieros (tanto del presupuesto nacional como de la economía familiar) y humanos (por ejemplo, de especialistas en geriatría) para satisfacer las necesidades de cuidados de salud de este grupo de la población. En particular, el incremento en la esperanza de vida, la mayor prevalencia de enfermedades crónicas y el aumento de los gastos de salud asociados con los adultos mayores significarán una mayor demanda de recursos financieros por parte de los sistemas sanitarios nacionales.

Los cambios en la estructura poblacional indican que para evitar el deterioro de las condiciones de vida y atender a las necesidades de salud del creciente número de adultos mayores se deberán aumentar sustancialmente el ahorro para la vejez y el financiamiento de los sistemas de salud. La ausencia o la insuficiencia de mecanismos de ahorro para satisfacer las necesidades de consumo y de salud de los adultos mayores obligarán a recurrir a transferencias -obligatorias o voluntarias - de la población en edad laboral a la población en edad de retiro. Sin estas transferencias, las condiciones de vida de los adultos mayores empeorarán.

\section{Dimensiones económicas del envejecimiento: sistemas de protección social y de ahorro para la vejez}

Una de las consecuencias del envejecimiento de la población será el incremento continuado de la tasa de ahorro interno. La prolongación del ciclo de 
vida implica que deben aumentar los ahorros para poder financiar las necesidades de consumo y de salud después del retiro (1). Este no parece ser el caso en la mayoría de los países de la Región, pues las tasas de ahorro interno en la década de 1990 permanecieron relativamente estables ${ }^{2}$ (2). Es decir, que para utilizar durante un mayor número de años las mismas tasas de ahorro para el retiro se deberá reducir el monto de las prestaciones y, por tanto, los niveles de vida de los adultos mayores se verán menoscabados, a menos que aumente el volumen de transferencias de las personas en edad laboral a las jubiladas.

Idealmente - si la información de que se dispone sobre el futuro es correcta y no hay costos de transacción ni fallas en el mercado-, la tasa de ahorro óptima durante la edad laboral es aquella que garantiza la satisfacción de las necesidades de consumo de bienes y servicios y de gastos en salud durante la vejez.

Desde el punto de vista de las políticas, existen pocas alternativas para corregir los errores de las decisiones (voluntarias) tomadas individualmente - fallas del mercado- con respecto a los ahorros necesarios para hacer frente a las necesidades de la vida durante la vejez. Estas son: la creación o expansión de sistemas de protección social y la creación de planes obligatorios (contributivos) de ahorro para la vejez mediante cuentas de capitalización individual (CCI).

La protección social puede estar organizada en sistemas de seguridad social (pilar 1), cuyo financiamiento proviene de contribuciones, o en sistemas de asistencia social financiados generalmente mediante impuestos (pilar 0). Según el tipo de prestación de pensiones, los primeros se pueden clasificar en contributivos de reparto (beneficios definidos), contributivos que garantizan una pensión mínima y universales (3). Los sistemas de ahorro para la vejez pueden estar basados en contribuciones obligatorias mediante CCI (pilar 2) o en contribuciones voluntarias (pilar 3$)^{3}$ (4). En ausencia de mecanismos de protección social y de planes de ahorro para la vejez, las necesidades de salud de los adultos mayores se deberán cubrir con transferencias intrafamiliares o mediante programas y actividades de caridad (pilar 4).

\footnotetext{
2 La tasa de ahorro - que agrupa las tasas de ahorro interno y externofue de cerca de $20 \%$ del producto interno bruto durante la década de 1990, por debajo de lo observado en la década anterior.

3 La referencia a los pilares 1, 2 y 3 sigue la terminología del Banco Mundial que ha sido aplicada en la caracterización de las reformas de la seguridad social.
}

\section{SISTEMAS DE PROTECCIÓN SOCIAL EN AMÉRICA LATINA Y EL CARIBE}

\section{Sistemas de seguridad social}

Entre los sistemas de protección social utilizados en la Región se encuentran los de la seguridad social (contributivos) que otorgan las prestaciones de pensiones de retiro mediante los programas de vejez, invalidez y muerte, y los de asistencia social (no contributivos) que otorgan prestaciones monetarias y no monetarias durante la vejez mediante los programas de asistencia social (cuadro 1). La mayoría de los sistemas de seguridad social de la Región funcionan mediante mecanismos de reparto, cuyo financiamiento proviene de las contribuciones que realizan los empleados y -en dependencia del país- de los empleadores y el gobierno. Los beneficios (prestaciones) o pensiones otorgadas se definen de acuerdo con la remuneración a la edad del retiro y el período de tiempo durante el cual el empleado contribuyó financieramente. Solo en Argentina y Canadá se aplica un sistema de seguridad social universal con programas que garantizan una pensión mínima a toda la población o a grandes segmentos de ella. En Argentina, este componente universal que forma parte de la pensión se denomina prestación básica universal (PBU) y se reajusta anualmente (5). En Canadá, el programa de seguridad para adultos mayores (old age security program, OASP) garantiza a la población un subsidio o pensión única que se otorga a todas las personas cuyos ingresos anuales sean inferiores a US\$ 35 000. El OASP funciona a la par del sistema contributivo de reparto que es parte de la seguridad social (obligatoria) (6).

Además de los sistemas de reparto, en la mayoría de los países de la Región existen esquemas de pensión mínima contributiva (cuadro 1). En Bermuda y Jamaica, la tasa fija o pensión básica fija se complementa con un componente que depende del tiempo de contribución del beneficiario. En el caso de Ecuador, además de las pensiones basadas en el reparto, se otorga una pensión mínima o reembolso a las personas cuyos ingresos o contribuciones sean insuficientes para ingresar al programa de pensiones. Finalmente, en Venezuela se otorga una pensión básica fija complementada por una suma que depende de los ingresos y una subvención adicional equivalente a $10 \%$ de los ingresos del beneficiario, todo como parte de un mismo sistema (7).

En varios países, el sistema de seguridad social basado en el reparto existe junto con programas de asistencia social (cuadro 1). Mediante estos programas se otorgan pensiones, subsidios o bonificaciones a los adultos mayores que se consi- 
CUADRO 1. Sistemas de seguridad social y programas de asistencia social en las Américas

\begin{tabular}{|c|c|c|c|c|}
\hline \multirow[b]{2}{*}{ Fase de aplicación } & \multicolumn{3}{|c|}{ Seguridad social } & \multirow{2}{*}{$\frac{\text { Asistencia social }}{\text { Pensión mínima no contributiva }^{b}}$} \\
\hline & Reparto $^{a}$ & Universal & Pensión mínima contributiva & \\
\hline En aplicación & $\begin{array}{l}\text { Antigua y Barbuda, Bahamas, } \\
\text { Barbados, Belice, Bermuda, } \\
\text { Brasil, Cuba, Dominica, Estados } \\
\text { Unidos de América, Granada, } \\
\text { Guatemala, Guyana, Haití, Islas } \\
\text { Vírgenes Británicas, Jamaica, } \\
\text { Panamá, Paraguay, Saint Kitts } \\
\text { y Nevis, San Vicente y las } \\
\text { Granadinas, Santa Lucía, } \\
\text { Trinidad y Tabago, Venezuela }\end{array}$ & Canadá & $\begin{array}{l}\text { Antigua y Barbuda, Bahamas, } \\
\text { Barbados, Belice, Bermuda, } \\
\text { Brasil, Canadá, Cuba, Dominica, } \\
\text { Estados Unidos de América, } \\
\text { Granada, Guyana, Haití, } \\
\text { Jamaica, Panamá, Saint Kitts } \\
\text { y Nevis, San Vicente y las } \\
\text { Granadinas, Santa Lucía, } \\
\text { Trinidad y Tabago, Venezuela }\end{array}$ & $\begin{array}{l}\text { Antigua y Barbuda, Bahamas, } \\
\text { Barbados, Belice, Bermuda, } \\
\text { Brasil, Canadá, Estados Unidos } \\
\text { de América, Saint Kitts y Nevis, } \\
\text { Trinidad y Tabago }\end{array}$ \\
\hline $\begin{array}{l}\text { Sistemas de } \mathrm{CCl} \\
\text { establecidos }\end{array}$ & $\begin{array}{l}\text { Argentina, Colombia, Costa } \\
\text { Rica, Perú }\end{array}$ & Argentina & $\begin{array}{l}\text { Colombia, Costa Rica, Méxicoc, } \\
\text { Perú }\end{array}$ & $\begin{array}{l}\text { Colombia, Costa Rica, Méxicoc, } \\
\text { Perú, Uruguay }\end{array}$ \\
\hline $\begin{array}{l}\text { Sistema } \mathrm{CCl} \text { en } \\
\text { fase de regulación } \\
\text { o puesta en } \\
\text { marcha }\end{array}$ & Ecuador, Honduras, Nicaragua & & Ecuador, Honduras, Nicaragua & Ecuador, Nicaragua \\
\hline $\begin{array}{l}\text { Sistemas de } \\
\text { reparto residuales }\end{array}$ & $\begin{array}{l}\text { Chile, El Salvador, Méxicoc } \\
\text { República Dominicana }\end{array}$ & & $\begin{array}{l}\text { Chile, El Salvador, República } \\
\text { Dominicana }\end{array}$ & Bolivia, Chile \\
\hline
\end{tabular}

Fuente: Administración para la Seguridad Social (6).

a Se refiere solamente a los programas de seguridad social del gobierno, no a los sistemas de seguridad social empresariales y gremiales.

${ }^{\mathrm{b}}$ En algunos casos se otorga mediante la prueba de necesidad. En Bolivia se otorga el BONOSOL.

${ }^{c}$ En México, el sistema de reparto se aplica solamente a los empleados y funcionarios del sector público.

deran indigentes, desvalidos o en condiciones de pobreza. ${ }^{4}$

\section{Sistemas de ahorro para la vejez}

A partir de la década de 1980 en varios países de la Región se crearon fondos de retiro mediante CCI. Este sistema de ahorro para la vejez puede ser de carácter obligatorio (pilar 2) o voluntario (pilar 3). En general, no es parte de los sistemas de protección social por consistir en cuentas de ahorro individual, ${ }^{5}$ aunque se ha aplicado en diversas modalidades, algunas de ellas en coexistencia con los sistemas de seguridad social preexistentes basados en el reparto (cuadro 1).

En la Región existen simultáneamente diferentes variantes de CCI (cuadro 2). Los países con un sistema único son aquellos donde la afiliación a las CCI es obligatoria. El antiguo programa de pen-

\footnotetext{
4 BONOSOL, en Bolivia, es uno de los programas más novedosos y otorga una suma anual a todas las personas mayores de 65 años. Este programa se financia con fondos del tesoro público provenientes de la privatización o venta de empresas públicas (8).

5 Para mayor información sobre este tema se puede consultar el anexo del capítulo 3 del Manual de estadística de las finanzas públicas, del Fondo Monetario Internacional (4).
}

siones de los sistemas de seguridad social basados en el reparto ha quedado desfasado y se mantiene solo para los antiguos contribuyentes que decidieron permanecer en él. ${ }^{6}$ Por su parte, los sistemas de CCI complementarios existen junto con los que lo precedieron y en algunos casos los beneficiarios pueden pasar de un sistema a otro (10). En los sistemas duales en competencia, el interesado debe elegir si coloca sus contribuciones obligatorias en un programa de la seguridad social o en una CCI.

En general, los esquemas de ahorro basados en CCI son financiados con los aportes obligatorios preestablecidos de acuerdo con los ingresos del empleado, del mismo modo en que se hacen los aportes a los programas de pensiones de los sistemas de seguridad social. Estas cuentas son capitalizables según el rendimiento del mercado. Al llegar a la edad del retiro, el empleado recibe una pensión en correspondencia con lo acumulado en su cuenta individual (contribución definida) gracias a lo aportado y al rendimiento del mercado. Estas cuentas son administradas por entidades creadas para ese fin (administradoras de fondos de pensiones) y funcionan bajo el control de alguna entidad del go-

6 Esta obligatoriedad es solo para los trabajadores que ingresan por primera vez al mercado laboral. 
CUADRO 2. Características de las cuentas de capitalización individual (CCI) en algunos países de la América Latina

\begin{tabular}{|c|c|c|c|c|}
\hline \multirow[b]{2}{*}{ País } & \multirow{2}{*}{ 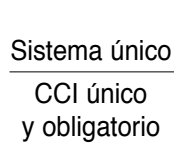 } & \multicolumn{2}{|c|}{ Sistema complementario } & \multirow{2}{*}{$\begin{array}{c}\text { Sistema dual } \\
\text { en competencia } \\
\text { Reparto o CCl } \\
\text { obligatorios }\end{array}$} \\
\hline & & $\begin{array}{l}\text { Reparto obligatorio } \\
\text { y CCl obligatoria }\end{array}$ & $\begin{array}{c}\text { Reparto obligatorio } \\
\text { y CCI voluntaria }\end{array}$ & \\
\hline Argentina & & & & $X$ \\
\hline Bolivia & $x^{a}$ & & & \\
\hline Colombia & & & & $X$ \\
\hline Costa Rica & & $X$ & & \\
\hline Chile & $\mathrm{X}$ & & & \\
\hline Ecuador $^{\mathrm{b}}$ & & $\mathrm{I} / \mathrm{R}^{\mathrm{a}}$ & & \\
\hline El Salvador & $\mathrm{X}$ & & & \\
\hline Honduras $^{c}$ & & & $\mathrm{l} / \mathrm{R}$ & \\
\hline Méxicod $^{d}$ & $X$ & & & \\
\hline Nicaragua $^{\mathrm{e}}$ & $\mathrm{l} / \mathrm{R}$ & & & \\
\hline Panamá & & & I/R & \\
\hline Perú & & & & $\mathrm{X}$ \\
\hline República Dominicana & $\mathrm{X}$ & & & \\
\hline Uruguay $^{\dagger}$ & & $X$ & & \\
\hline \multicolumn{5}{|c|}{$\begin{array}{l}\text { Fuente: Administración para la Seguridad Social (6) y Asociación Internacional de Organismos de Supervisión de Fondos de Pensiones (9). } \\
\text { a X: sistema que se aplica en el país; I/R: en fase de puesta en marcha o de regulación. } \\
\text { b La Ley } 2001-55 \text { de Seguridad Social que crea el sistema de cuentas individuales obligatorias aún no ha sido aplicada. } \\
\text { c El Decreto Legislativo } 319-2002 \text { que crea el régimen opcional complementario de AFP se encuentra en fase de puesta en vigor. } \\
\text { d Aplicable solo a los empleados del sector privado asegurados anteriormente por el Instituto Mexicano de Seguridad Social. } \\
\text { e El nuevo sistema se encuentra en fase de puesta en marcha. Será obligatorio para los afiliados al Instituto Nicaragüense de Seguridad Social } \\
\text { menores de } 43 \text { años de edad. } \\
\text { f La obligatoriedad de contribuir a la CCI depende de las escalas de ingresos. }\end{array}$} \\
\hline
\end{tabular}

bierno (superintendencias). En los sistemas privados de ahorro voluntario para la vejez, las fuentes de financiamiento de las CCI son los aportes voluntarios de los titulares de las cuentas de ahorro. Para promover el desarrollo de esquemas privados de ahorro voluntario, los gobiernos aplican incentivos, como exenciones tributarias a las cuentas de ahorro o a las CCI. ${ }^{7}$

\section{COBERTURA, GASTOS Y FINANCIAMIENTO DE LOS SISTEMAS DE PROTECCIÓN SOCIAL Y DE AHORRO PARA LA VEJEZ}

\section{Cobertura de los programas de pensiones: sistemas de seguridad social y de capitalización individual}

El tamaño y la importancia relativa de la población de adultos mayores, el nivel de desarrollo y la cobertura de los sistemas de protección social y

\footnotetext{
7 Un caso particular es el del programa Cuota Social en México, que funciona paralelo a las CCI. Esta contribución de monto único, calculada como un porcentaje del salario mínimo (actualmente 6,05\%), es aportada por el Estado y abonada diariamente a las CCI de todos los trabajadores que tengan su contribución al día. De esta manera funciona como un incentivo para que los trabajadores continúen aportando al CCI y a la vez introduce un mecanismo de prevención de la pobreza, ya que protege a los trabajadores de menores ingresos del pago de comisiones a las entidades administradoras (11).
}

de ahorro para la vejez varían considerablemente entre los países de la Región. ${ }^{8}$

Las personas mayores de 60 años representan una parte importante de la población total de América Latina y el Caribe. ¿Cuál es su situación de seguridad económica? ¿Cuántas de ellas se benefician o están amparadas por los programas de protección social? ¿Cuántas poseen CCI para garantizar su vejez?

En el cuadro 3 se presentan los datos estimados de la cobertura de los sistemas de protección social basados en pensiones de la seguridad social y de los sistemas de ahorro para la vejez. ${ }^{9}$ Estos datos revelan que en ciertos países de la Región, la mitad (50,1\%) de los adultos mayores de 65 años de edad cuenta con algún sistema de protección social de pensiones (pilar 1) o de ahorro para la vejez (pilar 2 ó 3).

La otra mitad de los adultos mayores en esos países no cuenta con ningún sistema de protección social o de ahorro (CCI) que le permita satisfacer

\footnotetext{
8 El envejecimiento de la población en los países de América Latina y el Caribe depende de la reducción en las tasas de fecundidad y mortalidad en cada país y del momento en que ocurre esa reducción.

9 El cuadro 3 recoge los indicadores de cobertura de los sistemas de protección social y de ahorro para la vejez publicados en anuarios estadísticos de organismos internacionales, informes administrativos nacionales, estudios y presentaciones sobre el tema de pensiones y sistemas de ahorro para la vejez. Aunque los datos no son totalmente comparables debido a la falta de uniformidad en los conceptos, métodos y fuentes de información utilizados, dan una idea general de la cobertura de los sistemas de protección social y de CCI.
} 
CUADRO 3. Porcentaje de adultos mayores de 65 años de edad con pensiones en la población, según el sistema y la fuente de información

\begin{tabular}{|c|c|c|c|c|}
\hline País & Año & $\begin{array}{l}\text { Sistema de } \\
\text { seguridad } \\
\text { social (SS) }\end{array}$ & $\begin{array}{c}\text { Cuentas de } \\
\text { capitalización } \\
\text { individual (CCl) }\end{array}$ & $\begin{array}{c}\text { Total } \\
(\mathrm{SS}+\mathrm{CCl})\end{array}$ \\
\hline \multirow[t]{3}{*}{ Argentina } & 1996 & & & $73,4^{\mathrm{a}}$ \\
\hline & 2001 & & & $68,7^{a}$ \\
\hline & 2002 & & & $66,2^{b}$ \\
\hline Bolivia & 2000 & & 11,9 & $11,9^{b}$ \\
\hline \multirow[t]{3}{*}{ Brasil } & 1992 & 74,0 & & $74,0^{c}$ \\
\hline & 1997 & 80,1 & & $80,1^{c}$ \\
\hline & 2002 & 81,5 & & $81,5^{c}$ \\
\hline \multirow[t]{4}{*}{ Chile } & 1996 & & & $50,8^{a}$ \\
\hline & 2000 & & & $48,5^{\mathrm{a}}$ \\
\hline & 2000 & & & $41,4^{b}$ \\
\hline & 2003 & 26,7 & 18,8 & $45,5^{c}$ \\
\hline Colombia & 1999 & & & $15,2^{b}$ \\
\hline \multirow[t]{2}{*}{ Costa Rica } & 2000 & & & $33,2^{b}$ \\
\hline & 2002 & & & $36,0^{c}$ \\
\hline \multirow[t]{2}{*}{ Ecuador } & 1994 & 12,2 & & $12,2^{\mathrm{a}}$ \\
\hline & 2001 & 11,6 & & $11,6^{a}$ \\
\hline \multirow[t]{5}{*}{ EI Salvador } & 1998 & 8,8 & & $8,8^{b}$ \\
\hline & 2000 & 13,5 & 0,0 & $13,6^{c}$ \\
\hline & 2001 & 14,2 & 0,1 & $14,3^{c}$ \\
\hline & 2002 & 15,6 & 0,2 & $15,8^{c}$ \\
\hline & 2003 & 15,7 & 0,5 & $16,2^{\mathrm{c}}$ \\
\hline \multirow[t]{3}{*}{ México } & 1996 & 17,9 & & $17,9^{a}$ \\
\hline & 2001 & & & $19,0^{\mathrm{a}}$ \\
\hline & 2001 & & & $20,0^{b}$ \\
\hline \multirow[t]{3}{*}{ Perú } & 1999 & & & $19,5^{b}$ \\
\hline & 2000 & & & $23,5^{\mathrm{b}}$ \\
\hline & 2000 & & & $37,2^{\mathrm{a}}$ \\
\hline \multicolumn{5}{|l|}{ República } \\
\hline Dominicana & 1997 & & & $14,6^{b}$ \\
\hline Venezuela & 2003 & 39,0 & & $39,0^{c}$ \\
\hline
\end{tabular}

a Datos correspondientes al período entre 1994 y 2002. Estimados obtenidos de la base de datos de Encuestas de Hogares de los países (11).

${ }^{b}$ Datos correspondientes al período entre 1997 y 2000. Estimados derivados de las Encuestas de Hogares (6).

c Datos correspondientes a la población mayor de 60 años. Estimados de la OPS basados en registros administrativos (1).

sus necesidades de consumo y de salud durante la vejez. Las necesidades de estas personas se cubren mediante activos acumulados durante la edad laboral, transferencias familiares intergeneracionales o programas asistenciales e instituciones de beneficencia (pilar 4).

Las reformas de los programas de pensiones de la seguridad social y la creación de sistemas de ahorro para la vejez mediante CCI no han logrado garantizar que la población cuente con los recursos suficientes para afrontar las necesidades de consumo y de salud durante la vejez después de jubilarse (cuadro 4). Se debe tener en cuenta que no todos los afiliados al sistema de CCI cumplen con sus contribuciones a las cuentas de ahorro individual, lo que se refleja en la diferencia que se observa entre la proporción de afiliados y de contribuyentes
CUADRO 4. Población económicamente activa (PEA) a con cuentas de capitalización individual. Diciembre de 2003

\begin{tabular}{lccc}
\hline \multicolumn{1}{c}{ País } & $\begin{array}{c}\text { Afiliados/ } \\
\text { PEA }\end{array}$ & $\begin{array}{c}\text { Contribuyentes/ } \\
\text { PEA }^{\text {a }}\end{array}$ & $\begin{array}{c}\text { Contribuyentes/ } \\
\text { Afiliados }\end{array}$ \\
\hline Argentina & 43,3 & 15,3 & 35,2 \\
Bolivia & 18,6 & 7,2 & 39,0 \\
Chile & 74,8 & 38,8 & 51,9 \\
Colombia & 20,4 & 9,9 & 48,7 \\
Costa Rica & 47,9 & 35,0 & 73,1 \\
El Salvador & 29,9 & 13,9 & 46,3 \\
México & 53,0 & 20,8 & 39,3 \\
Perú & 21,1 & 8,8 & 41,9 \\
República & & & \\
$\quad$ Dominicana & 16,5 & 13,7 & 82,8 \\
Uruguay & 32,8 & 17,3 & 52,7 \\
\hline
\end{tabular}

Fuentes: Datos de la Asociación Internacional de Organismos de Supervisión de Fondos de Pensiones (AIOS) (9) y CEPAL (1).

a Población de 15 a 59 años de edad en el año 2000.

en la población económicamente activa (PEA) ${ }^{10}$ Los afiliados a estos sistemas que no cotizan no contarán con los fondos necesarios que les garantizarían recibir la pensión mínima después de la edad del retiro, o sus ahorros solo les permitirán cubrir la pensión de retiro mínima garantizada por otros fondos después de contribuir con un monto o un número de años determinados. Esta situación es más crítica en los países donde la afiliación al sistema de CCI es obligatoria para los trabajadores que ingresan por primera vez al mercado laboral, como Chile, Bolivia, El Salvador y la República Dominicana. ${ }^{11}$

La proporción de personas mayores de 65 años de edad que declaran recibir algún tipo de pensión o jubilación difiere considerablemente de un país a otro (figura 2). En el extremo inferior se encuentra Nicaragua, con una cobertura de 6,3\%, mientras que en el límite superior está Brasil con una cobertura de $85,4 \%$. Ambos países tienen sistemas de seguridad social por reparto.

En cuanto a la tendencia de los niveles de cobertura de ambos sistemas, los resultados son heterogéneos. En las dos últimas décadas, las reformas de la seguridad social han promovido la transformación de los sistemas de protección social en esquemas de ahorro basados en CCI $(12,13)$. Sin embargo, aun en la mayoría de los países de la Región predominan los programas de pensiones basados en sistemas de seguridad social, como los de re-

${ }^{10}$ Ello se desprende de la relación entre el número de contribuyentes y el número de afiliados, que varía de $35,2 \%$ en la Argentina a $82,8 \%$ en República Dominicana, que fue el último país en adoptar el sistema CCI.

11 En México, la obligatoriedad afecta solamente a los empleados dependientes del sector privado, por lo que este dato no refleja la situación de todos los adultos mayores. 
FIGURA 2. Personas mayores de 65 años de edad que declararon recibir pensión o jubilación ${ }^{\mathrm{a}}$

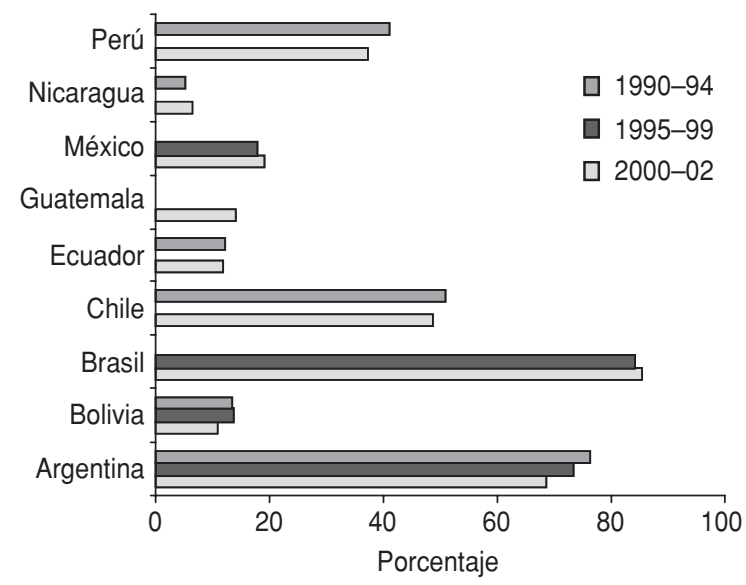

Fuente: Oficina Internacional del Trabajo (11).

a Datos de la base de datos de las Encuestas de Hogares de los Países (Argentina: 1992, 1996 y 2001; Bolivia: 1997,1999 y 2002; Brasil: 1995 y 2001; Chile: 1996 y 2000; Ecuador: 1994 y 1998; Guatemala: 2000; México: 1996 y 2000; Nicaragua: 1993 y 2001; Perú: 1994 y 2000).

parto de beneficios definidos. ${ }^{12}$ Entre los períodos 1990-1999 y 2000-2002, solo tres países (Nicaragua, México y Brasil) mejoraron sus niveles de cobertura, mientras que en Perú, Ecuador, Chile, Bolivia y Argentina - países que con la excepción de Ecuador han adoptado el sistema de ahorro para la vejez- se observó una tendencia negativa (figura 2). Esto hace que un amplio sector de la población en edad laboral permanezca desprotegido después de su retiro, principalmente en los países donde las CCI son la única opción disponible para los nuevos trabajadores.

En resumen, las reformas de los programas de pensiones de los sistemas de seguridad social y su transformación en sistemas de CCI no se complementaron con programas de extensión de la protección social que permitieran hacer frente a las necesidades de consumo y de salud durante la vejez. En la práctica, estas reformas llevaron al desmantelamiento de los sistemas de protección social para la vejez basados en la seguridad social, sin crear mecanismos alternativos.

Ante la ausencia de sistemas públicos de protección social, el avance y las posibilidades de desarrollo de los esquemas de ahorro basados en CCI, la baja cobertura de los programas de protección social y el alcance limitado de los programas de CCI

\footnotetext{
12 En Brasil, donde se mantiene el sistema de seguridad social, las reformas introducidas entre 1995 y 2003 modificaron los parámetros de los beneficios (reforma "paramétrica") con el aumento de la edad de retiro y la extensión de la duración de los beneficios. Posteriormente se estableció un esquema complementario de pensiones y se homogenizaron las reglas para los trabajadores de los sectores público y privado $(14,15)$.
}

—agravado por la proporción de afiliados que no contribuyen a estos sistemas- en los próximos 25 años se prevén grandes dificultades para los adultos mayores de América Latina ${ }^{13}(16,17)$. Aunque en este contexto las transferencias intergeneracionales desempeñarán un papel clave, el resultado puede llegar a ser muy grave si se tiene en cuenta el incremento substancial que se observará en la tasa de dependencia económica de los adultos mayores (figura 1).

\section{Prestaciones de los sistemas de protección social y de ahorro para la vejez}

Además de las diferencias observadas entre los niveles de cobertura de la población en los diferentes países, también hay diferencias importantes en cuanto a las prestaciones - es decir, el importe de las pensiones-que reciben los adultos mayores beneficiarios de los programas de protección social para la vejez y de los esquemas de ahorro privado basados en CCI. Estas diferencias también se manifiestan entre los diferentes grupos de población de un mismo país.

El monto promedio de las pensiones recibidas mensualmente por los beneficiarios de estos programas varían entre US\$ 78,00 en Perú y US\$ 81,00 en Venezuela, a cerca de US\$ 500,00 en Chile y Costa Rica (cuadro 5). Estos datos revelan que además de las diferencias en la cobertura, existen diferencias igualmente marcadas con respecto a la cuantía de esos beneficios en América Latina.

Asimismo, dentro de los países hay grandes desigualdades en cuanto al importe promedio de las pensiones que reciben los beneficiarios de los sistemas de seguridad social. En algunos países de la Región, los beneficios de algunos grupos de pensionados son ligeramente superiores a US\$2,00 diarios, que es el ingreso utilizado por el Banco Mundial para definir la pobreza. Por otro lado, las prestaciones mensuales que reciben los beneficiarios de los programas de pensiones de Argentina, Brasil, Chile, Costa Rica y Panamá (alrededor de US\$ 500,00 ) sitúan a este grupo de la población alrededor de o por encima del ingreso promedio por habitante en esos países.

En el caso de Brasil, el monto promedio de las pensiones mensuales de los jubilados del sector público muestra grandes diferencias entre los diferentes grupos, según su categoría ocupacional (cuadro 6). En el año 2002 en un extremo se encontraban los jueces federales, con pensiones de alrededor de

\footnotetext{
13 En un libro publicado recientemente por el Banco Mundial se afirma que en América Latina el primer pilar de prevención de la pobreza debería ser el que haya captado mayor atención en la última década (5).
} 
CUADRO 5. Gasto per capita en pensiones para personas mayores de 60 años que cuentan con algún tipo de cobertura. Año 2000

\begin{tabular}{|c|c|c|c|c|}
\hline \multirow[b]{2}{*}{ País } & \multirow{2}{*}{$\begin{array}{l}\text { Porcentaje de población mayor } \\
\text { de } 60 \text { años con cobertura }\end{array}$} & \multirow{2}{*}{$\begin{array}{c}\text { Gasto anual en } \\
\text { pensiones, \% del PIB }\end{array}$} & \multicolumn{2}{|c|}{ Gasto per capita en población cubierta } \\
\hline & & & US\$/día & US\$/mes \\
\hline Argentina & 66,2 & 6,2 & 15 & 450 \\
\hline Bolivia & 11,9 & 2,5 & 9 & 273 \\
\hline Brasil & 81,5 & 4,9 & 7 & 225 \\
\hline Chile & 45,5 & 5,8 & 17 & 513 \\
\hline Colombia & 15,2 & 1,1 & 6 & 174 \\
\hline Costa Rica & 36,0 & 3,8 & 16 & 472 \\
\hline Ecuador & 11,6 & 1 & 4 & 131 \\
\hline El Salvador & 16,2 & 1,3 & 6 & 194 \\
\hline México & 19,0 & 0,4 & 5 & 149 \\
\hline Perú & 37,2 & 1,2 & 3 & 78 \\
\hline Venezuela & 39,0 & 0,5 & 3 & 81 \\
\hline
\end{tabular}

Fuente: Datos tomados de Organización Internacional del Trabajo (18), Organización de Naciones Unidas (19), US Census Bureau (20) y CEPAL (1).

a Los datos de PIB y población corresponden al año 2000. Los datos de cobertura corresponden al valor más reciente del cuadro 3.

${ }^{b}$ El gasto anual en pensiones en porcentaje del PIB corresponde al período 1996-2000 (21).

CUADRO 6. Diferencias entre el promedio mensual de prestaciones de Brasil y Méxicoa

\begin{tabular}{|c|c|c|c|c|c|}
\hline \multirow[b]{2}{*}{ Categoría } & \multicolumn{2}{|c|}{ Brasil, 2002} & \multirow[b]{2}{*}{ Categoría } & \multicolumn{2}{|c|}{ México, 2002} \\
\hline & $\begin{array}{l}\text { Número de } \\
\text { pensionados } \\
\text { (miles) }\end{array}$ & $\begin{array}{l}\text { Pensión mes } \\
\text { promedio } \\
\text { (US\$) }\end{array}$ & & $\begin{array}{l}\text { Número de } \\
\text { pensionados } \\
\text { (miles) }\end{array}$ & $\begin{array}{l}\text { Pensión mes } \\
\text { promedio } \\
\text { (US\$) }\end{array}$ \\
\hline Trabajadores civiles federales & & & IMSS, empleados & 2034 & 172 \\
\hline (RPPS) & 940 & & IMSS, empleadores & 121 & 1784 \\
\hline Poder ejecutivo & & 944 & ISSSTE & 442 & 439 \\
\hline Jueces federales & & 5157 & ISSFAM & 45 & \\
\hline Banco central & & 2897 & PEMEX & 65 & 1304 \\
\hline Fuerzas armadas & & 1750 & CFE & 20 & 1459 \\
\hline Poder legislativo & & 3030 & Compañía de luz y fuerza & & \\
\hline Poder judicial & & 3177 & del centro & 18 & 2292 \\
\hline Empleados privados (RGPS) & 21200 & & Estados y municipios & & \\
\hline Por edad & & 310 & Banca de desarrollo & 4 & \\
\hline Por tiempo de servicio & & 101 & Universidades & 8 & \\
\hline Promedio RGPS & & 157 & & & \\
\hline
\end{tabular}

US\$ 5 000,00 mensuales en promedio, y los pensionados del Banco Central y de los de los poderes legislativo y judicial, con una pensión promedio de aproximadamente US\$ 3 000,00 mensuales. Mientras, la pensión mensual promedio de los empleados del sector privado fue solamente de US\$ 310,00 y la de los empleados privados del sistema de seguridad social, de aproximadamente US\$157,00.

En México se observan desigualdades similares entre el promedio de las pensiones recibidas por los empleados del sector público y los del sector privado beneficiarios de los diferentes sistemas de seguridad social (18). El monto promedio mensual de las pensiones de los beneficiarios del Instituto de Seguridad Social de los Trabajadores del Estado (ISSTE) fue de US\$ 430,00, mucho menos que lo recibido mensualmente por los pensionados de la Empresa Nacional de Petróleo de México (PEMEX) y de la Comisión Federal de Electricidad (CFE) (US\$ 1 300,00) y de la Compañía de Luz y Fuerza 
del Centro (US\$ 2 200,00). En el caso de los empleados del sector privado beneficiarios de los programas de pensiones del Instituto Mexicano de Seguridad Social (IMSS), el monto promedio de las pensiones de los empleados - aproximadamente el 95\% de los beneficiarios del programa- fue mucho menor (US\$ 170,00 mensuales) que el recibido por los empleadores (US\$ 1700,00 mensuales), que constituían el $5 \%$ de los beneficiarios de ese programa (cuadro 6).

\section{PROGRAMAS DE PROTECCIÓN SOCIAL Y DESIGUALDADES SOCIALES: ASPECTOS MACROECONÓMICOS Y DESAFÍOS DE LAS POLÍTICAS}

Como se puede observar, independientemente de la manera en que se organicen los sistemas de protección social o de ahorro para la vejez, los esquemas de ahorro de los programas de capitalización y los mecanismos de financiamiento de los sistemas de protección social no garantizan el ahorro o las transferencias necesarios para satisfacer las necesidades de consumo de los adultos mayores en muchos países de la Región.

El incremento del número de adultos mayores plantea nuevas dificultades para el diseño de las políticas sociales dirigidas a atender a las necesidades de este grupo de la población. La ausencia o la baja cobertura de los sistemas de protección social para la vejez y de capitalización individual indican que la carga de la demanda de recursos para satisfacer las necesidades de consumo y de salud de los adultos mayores recaerá sobre los gobiernos y las familias.

En el caso de los gobiernos, esta demanda se manifestará en la exigencia de extender la cobertura de los sistemas de seguridad social y de aplicar o ampliar los programas de protección social para los adultos mayores. En el caso de las familias, esta demanda se traducirá en transferencias intergeneracionales de la población económicamente activa hacia los adultos mayores que no estén cubiertos por algún sistema de protección social o cuya cobertura sea insuficiente para mantener un nivel de vida satisfactorio. Estas transferencias podrán realizarse de manera obligatoria - mediante el aumento de impuestos para financiar los programas de protección social— o por transferencias voluntarias dirigidas a las personas de mayor edad. Cualquiera de estos esquemas llevará a la reducción de los ingresos de la actual población laboralmente activa y por tanto a una reducción de la tasa de ahorro, lo que afectará negativamente a la tasa de crecimiento de las economías nacionales.
A pesar de la abundante información disponible sobre las fallas del mercado y las insuficiencias de las decisiones individuales para garantizar niveles adecuados de ahorro para la vejez, varios países de la Región han puesto en marcha reformas que fomentan el desarrollo de mercados privados de pensiones. El resultado económico de estas reformas ha sido la reducción de la carga fiscal actual y la promoción del desarrollo de instituciones de intermediación financiera y del mercado de capitales. Sin embargo, el impacto de la contribución a los sistemas de protección social en el crecimiento económico y en la reducción del costo de la mano de obra para el empleador (formado por el salario y los beneficios) no ha sido tan elevado. Asimismo, el incremento de la cobertura de los programas de capitalización individual no ha logrado compensar la reducción de la cobertura de los sistemas de protección social, lo que ha dado como resultado la reducción del número de personas con algún sistema de protección de ingresos para la vejez. ${ }^{14}$

El mayor desafío que enfrentan los responsables de elaborar las políticas y de tomar decisiones en los países de América Latina y el Caribe es corregir las "fallas de los gobiernos" y aplicar políticas que permitan subsanar las "fallas del mercado" que han impedido alcanzar la tasa de ahorro necesaria (óptima) mediante decisiones individuales. De lo contrario, la población laboralmente activa de hoy tendrá una vejez en condiciones de pobreza, sin los recursos suficientes para satisfacer sus necesidades de consumo y de salud.

\section{SYNOPSIS}

\section{Social protection systems for older adults in Latin America and the Caribbean}

Only one half (50.1\%) of all older adults in Latin America currently receive benefits under some form of social protection system for the elderly, either through social assistance or social security programs, or have access to a savings plan for old age in the form of individual capitalization accounts (ICA) based on mandatory or voluntary contributions. The other half enjoys no social protection at all; its consumer and health needs are covered through accrued assets, voluntary family transfers, or charitable deeds. Social security pension plan reforms and the creation of social-security-based savings plans for old age in the form of ICA have failed to yield the expected results in terms of coverage and benefits. Ove-

\footnotetext{
14 Con la excepción de Chile, donde alrededor de 50\% de la fuerza laboral se encurentra afiliada a sistemas de capitalización individual, la cobertura de estos sistemas no llega a $20 \%$ de la fuerza laboral.
} 
rall, reforms have led to the dismantling of social-securitybased social protection systems for the elderly without creating alternative mechanisms for social protection. This document describes the various social protection systems and savings plans for old age that are in place in countries of the Region and looks at current challenges in terms of correcting "government failures" and applying policies that will redress "market failures," so that the working population can count on having enough resources to satisfy its consumer and health needs after retirement.

Key words: social security, pensions, demographic transition, aged, Latin America, Caribbean Region.

\section{REFERENCIAS}

1. Comisión Económica para América Latina y el Caribe. América Latina y el Caribe: el envejecimiento de la población 1950-2050. Boletín Demográfico. 2003;(72). Hallado en: http://www. eclac.org/cgi-bin/getProd.asp? $\mathrm{xml}=/$ publicaciones $/ \mathrm{xml} / 1 / 13371 /$ P13371.xml\&xsl=/celade/tpl/p9f.xsl\& base $=/$ tpl/top-bottom.xslt. Acceso el 15 de abril de 2005.

2. International Monetary Fund. How will demographic change affect the global economy? En: International Monetary Fund. World economic outlook. The global demographic transition. Washington, D.C.: IMF; 2004. Pp. 137-80. Hallado en: http://www.imf.org/external/pubs/ft/ weo/2004/02/. Acceso el 15 de abril de 2005.

3. Economic Commission for Latin America and the Caribbean. Growth with stability. Financing for development in the new international context (up-dated version). Santiago, Chile: ECLAC; 2002. Pp. 49-50. (Libros de la CEPAL No. 67).

4. Fondo Monetario Internacional. Manual de Estadísticas de Finanzas Públicas 2001. Washington, D.C.: FMI; 2002. Hallado en: http:/ / www.imf.org/external/ pubs/ft/gfs/manual/esl/pdf/ch2ann. pdf. Acceso el 15 de abril de 2005.

5. Gill I, Packard T, Yermo J. Keeping the promise of social security in Latin America. Washington, D.C.: The World Bank, Stanford University Press; 2005.

6. Social Security Administration. Social security programs throughout the world: the Americas, 2003. Washington, D.C.: SSA; 2003. Hallado en: http:// www.ssa.gov/policy/docs/progdesc/ ssptw/2002-2003/americas/index. html. Acceso el 15 de abril de 2005.

7. Canada Pension Plan. Annual report of the Canada Pension Plan, 2001-2002. Hull, Québec: Human Resource Development Canada; 2002. Hallado en: http://www.sdc.gc.ca/en/isp/pub/ cpp/report/2002/annrpt2002.pdf. Acceso el 15 de abril de 2005.
8. Pugatch T. BONOSOL: Bolivia's universal pension program. En: Gill I, Packard $\mathrm{T}$, Yermo J. Keeping the promise of social security in Latin America. Washington, D.C.: The World Bank, Stanford University Press; 2005. Pp. 210-1.

9. Asociación Internacional de Organismos de Supervisión de Fondos de Pensiones. La capitalización individual en los sistemas provisionales de América Latina. Santiago, Chile: AIOS; 2003. Hallado en: http://www.aiosfp.org/documentos/ libro.pdf. Acceso el 15 de abril de 2005.

10. Pugatch T. The social quota: preventing poverty among the elderly in Mexico. En: Gill I, Packard T, Yermo J. Keeping the promise of social security in Latin America. Washington, D.C.: The World Bank, Stanford University Press; 2005. Pp. 208-9.

11. Oficina Internacional del Trabajo. Panorama laboral, 2003. Lima: OIT; 2003. Hallado en: http://www.oit.org. pe/portal / d ocumentos / panor a ma laboral_2003_texto_completo.pdf. Acceso el 15 de abril de 2005.

12. Larrain A. Privatizing social security in Latin America. Dallas, Texas: National Center for Policy Analysis; 1999. (NCPA Policy Report No. 221). Hallado en: http:/ / www.ncpa.org/studies/s221. html. Acceso el 15 de abril de 2005.

13. Bertranou F. Lecciones y desafíos de 23 años de reformas estructurales de los sistemas de jubilaciones y pensiones en América Latina [presentación en congreso]. Ponencias del Seminario Internacional El Futuro de la Previsión Social en Argentina y el Mundo: Evaluación y Desafíos; 2004 abril 20, Buenos Aires, Argentina. Santiago, Chile: Oficina Internacional del Trabajo; 2004. Pp. 43-56.

14. Médici A. The political economy of reform in Brazil's civil servant pension scheme. Washington, D.C.: InterAmerican Development Bank; 2004 (Technical Note on Pensions No. 002).
15. Schwarzer H, Da Silva de Paiva LH, Ferreira de Santana RL. Cobertura previdenciária: evolução 1999-2002 e aperfeiçoamento metodológico. Informe de Previdência Social. Vol. 16. Brasilia: Ministerio da Previdência Social; 2004.

16. Gill I, Packard T, Yermo J. Rethinking social security priorities in Latin America. En: Keeping the promise of social security in Latin America. Washington, D.C.: The World Bank, Stanford University Press; 2005. Pp. 1-15.

17. Economics focus: oversold. The World Bank changes its tune on Latin America's privatized pensions. The Economist. 23 de septiembre de 2004.

18. International Labor Organization. Cost of social security 1990-1996. Statistical tables by country. Geneva: ILO; 1997. Hallado en: http://www.ilo.org/public/english/ protection/socsec/publ/css/cssindex. htm. Acceso el 15 de abril de 2005.

19. United Nations, Statistic Division. National accounts main aggregates. New York: United Nations; 2004. Hallado en: http://unstats.un.org/unsd/snaama/ Introduction.asp. Acceso el 15 de abril de 2005. (Basic data selection).

20. United States Census Bureau. Summary demographic data. International database. Washington, D.C.: US Census Bureau; 2003. Hallado en: http://www. census.gov/ipc/www/idbnew.html. Acceso el 15 de abril de 2005.

21. Engler T. Economía del envejecimiento exitoso [presentación en seminario]. Seminario Envejecimiento Poblacional y Seguridad Social; 2004 octubre 11-15, México, D.F, México. México, D.F.: Centro Interamericano de Estudios de Seguridad Social; 2004.

22. Raya MA. Pensiones: elementos a considerar en la agenda legislativa [presentación en Internet]. México, D.F.; 2004. Hallado en: http://www.diputados.gob. $\mathrm{mx} /$ comisiones59legislatura/seguridad social/docts/pensiones.pdf. Acceso el 15 de abril de 2005. 